\title{
SCHULDRAMEN UND ORATORIEN BEI DEN PRESSBURGER JESUITEN IM 18. JAHRHUNDERT
}

Das Jesuitenkollegium in Preßburg war nicht das größte und wichtigste in der Slowakei, größer und bedeutender war bestimmt jenes in Tyrnau (heute Trnava), inklusive der 1635 gegründeten Universität, nach Wien überhaupt das zweitwichtigste Jesuitenhaus der großen, von Passau bis nach Ungvár (heute Užhorod in der Ukraine) reichenden Provincia Austriae Societatis Jesu. ${ }^{1}$ Das Preßburger Kollegium $^{2}$ schöpfte aber daraus, dass Preßburg in der Zeit der türkischen Besetzung eines großen Teils des Königreichs Ungarn die Hauptstadt des Landes war und hier z. B. der Primas von Gran (Esztergom) residierte; während das Graner Kapitel nach Tyrnau übersiedelte, hatten die Erzbischöfe ihre Residenz in Preßburg.

Deshalb war auch das Niveau der Musikpflege und des Schuldramas bei den Preßburger Jesuiten mit jenem in Tyrnau durchaus vergleichbar, bzw. teilweise noch höher. Es ist kein Zufall, dass eben in Preßburg das Drama Filius viva imago anlässlich der Krönung von Joseph I. (1688) mit Musik des Wiener Hoforganisten Ferdinand Tobias Richter (1651-1711), des wahrscheinlich prominentesten und besten Komponisten der Musik zum jesuitischen Drama im österreichischmitteleuropäischen Raum im 17. Jahrhundert, ${ }^{3}$ aufgeführt wurde.

1 Zur Geschichte der Jesuiten in der Slowakei vgl. z. B. KRAPKA, Emil SJ - MIKULA, Vojtech SJ. Dejiny Spoločnosti Ježišovej na Slovensku 1561-1988. 1. Ed. Cambridge, On. (Canada): Dobrá kniha 1990.

2 Zur Geschichte des Preßburger Jesuitenkollegiums ibid., S. 105-110. Nach dem Aufenthalt der ersten jesuitischen Missionare in Preßburg (1566-1570) wurde von P. Pázmány (1623) bei der Kirche St. Martin in der Kapitelgasse eine Residenz mit Schule gegründet, die 1630 zum Kollegium erhoben wurde. 1642 wurde das neue Collegium Emericianum gegründet, 1672 wurde das Jesuitenkollegium in das ehem. Ev. a. v. Kollegium mit der St. SalvatorKirche (ehem. Kirche der hl. Dreifaltigkeit) am Hauptplatz übersiedelt, in der Kapitelgasse blieb seither nur eine Residenz (bis 1773).

Zum Jesuitendrama in Wien und Tobias Ferdinand Richter siehe näher KRAMER, Waltraute. Die Musik im Wiener Jesuitendrama von 1677 bis 1767 (Diss.), Wien 1965. Zum Drama Filius viva imago (1688) vgl. STAUD, Géza. A Maygarországi jezsuita iskolai színjátékok forrásai (Fontes ludorum scenicorum in scholis S. J. Hungariae), Bd. I, Budapest: Magyar Tudományos Akadémia 1984, S. 378-379. 
Die ältesten schriftlichen Angaben über die Musik bei den Theateraufführungen in Preßburg stammen nach G. Staud aus dem Jahr 1642. ${ }^{4}$ Schon im 17. Jahrhundert wurde außer in Latein auch in Deutsch und Slowakisch gespielt, vor allem während der Prozessionen in der Stadt oder zum Kalvarienberg.

Das hohe Niveau hatten die Jesuitendramen und die musikdramatischen Gattungen insgesamt in Preßburg vor allem im 18. Jahrhundert. Im Preßbruger Jesuitenkollegium und in der Salvator-Kirche wurden nämlich nicht nur die gewöhnlichen Schuldramen, sondern auch Oratorien und andere musikdramatische Gattungen aufgeführt. Aus dieser Hinsicht stellt also Preßburg eher eine Ausnahme in der Slowakei dar. Zwar wurden manchmal die komplett vertonten Dramentexte auch in Tyrnau, ja sogar in kleineren Kollegien, wie z. B. in Komárno aufgeführt, in Tyrnau z. B. das 1749 aufgeführte Hl. Johann von Nepomuk-Drama Inocentia laesa, in Komárno wiederum 1748 - anlässlich der Grundsteinlegung der neuen Kirche - ein näher nicht bekanntes ,ad formam Italicae operae“ aufgeführtes Drama, ${ }^{5}$ dies waren jedoch eher Ausnahmen. Das „goldene Zeitalter“ des Schuldramas und des Oratoriums im Preßburger Jesuitenkollegium, sowie in der jesuitischen Salvator-Kirche waren vor allem die 20er und 30er Jahre des 18. Jahrhunderts, als in Preßburg eine ausgezeichnete Musikkapelle des Fürstprimas Emericus Esterházy wirkte.

Im 18. Jahrhundert stieg auch die Zahl der Aufführungen beachtlich: z. B. 1729 waren es 6 von verschiedenen Klassen aufgeführte Schuldramen (inklusive eines Drama majus), alle mit Musik, 1730 waren alle 4 Actiones majores (neben 4 weiteren Deklamationen) auch mit Musik, 1731 waren es sogar insgesamt 9 Theateraufführungen usw.:

\section{Die Zahlen der Theateraufführungen bei den Preßburger Jesuiten in den 20er und 30er Jahren}

(nach G. Staud: Fontes ludorum scenicorum, Bd. I, S. 365ff)

$\begin{array}{lll}1725-6 & 1730-8 & 1738-7 \\ 1726-4 & 1731-9 & \text { etc. } \\ 1727-2 & 1732-4 & \\ 1728-6 & 1733-1(?) & \\ 1729-6 & 1734-5 & \end{array}$

4 STAUD, Géza. op. cit., Bd. I, S. $365 \mathrm{ff}$.

5 Ibid., Bd. I, S. 192. Innocentia laesa ist eigentlich kein „Jesuitendrama“, es handelt sich um ein komplett vertontes und anlässlich der Doktorpromotion des Pauliners P. Stephanus Péchy in der Tyrnauer Universitätskirche aufgeführtes Drama - vgl. dazu KAČIC, Ladislav. Die Musik der Jesuitendramen in der Slowakei (1600-1773). Ein Beitrag zur Geschichte der Provincia Austriae SJ. In Ethnologische, historische und systematische Musikwissenschaft (Oskár Elschek zum 65. Geburtstag). Ed. Franz Födermayr - Ladislav Burlas. Bratislava: ASCO Art and Science 1998, S. 324.); zu Komárno vgl. STAUD, Géza, op. cit., S. 488. 
Die meisten Schuldramen waren mit Musik (und Tanz): in allen zwischen 1729 und 1731 gedruckten Periochen der Preßburger Jesuitendramen gibt es wenigstens allgemeine Angaben über die Musik (wie z. B. „His accedunt Musici, Saltatores, Ephebi, Milites“ u. ä.): Clitus (1729), Haldanus (1729), Fortitudo Christiana (1729), Ramirum inter et Garsiam Germanos pietas mutua (1730), Craterus (1730), Juventus tenera (1730), Remanedus (1730), Cleonymi insignis in parentum pietas (1731), Rara concordia fratrum (1731), Joannes Kiu (1731), Triumphus christianitatis in Floribundo (1731); es gab zu dieser Zeit in Preßburg nur ausnahmsweise Dramenvorstellungen ohne Musik, z. B. Zrinius ad Szighe$\operatorname{tum}(1738)^{6}{ }^{6}$

Wenn auch die Musikkapelle der Preßburger Jesuiten regelmäßig gut besetzt war $^{7}$ - es wirkten hier viele gute Musiker mit, wie z. B. der langjährige Regenschori G. Engel, sowie seine Nachfolger Richter und L. Popp, die Organisten Fr. Šípek und W. Löw, der ausgezeichnete Tenorist A. Renner, des weiteren J. P. Fux, oder F. Fauner, F. Kurz, J. Vachovský und andere Studenten des Kollegiums (dazu kamen regelmäßig noch die Stadttrompeter usw.) -, nahmen an den größeren Schuldramenaufführungen manchmal auch andere Musiker und Künstler teil, z. B. jene aus der Fürstbischöflichen Hofkapelle.

Sehr gut sind wir - vor allem dank Forschungen von Prof. I. Kilián ${ }^{8}$ - über eine solche prachtvolle Dramenaufführung, an der Musiker der Fürstbischöflichen Hofkapelle mitwirkten, informiert: Ludus Fortunae Bajazete a Tamerlane devictus (1734). Im Diarium des Preßburger Jesuitenkollegiums (Diarium Scholasticum Cardinalitii S. J. Gymnasii Posonii ad S. Salvatorem inchoatum Anno 1732) ist der ganze Verlauf der Vorbereitungen (alle Proben usw.), sowie der Dramenaufführung selbst gründlich und detailliert beschrieben.

Am 2. August reiste der Präfekt des Preßburger Kollegiums nach Tyrnau, um M. Leibetzeder, den Komponisten, nach Preßburg zu begleiten; am 4. August kehrten die beiden zurück (alle folgende Zitate aus dem Diarium sind in Kursivschrift):

\section{Aug. 1734 abivit P. Praefectus Tyrnaviam pro Magistro Leibeczeter Composi-} tore musices.

4. Aug. Redivit P. Praefectus cum Magistro Musices Tyrnaviâ.

Am 6. August begannen nach der Vesper die Proben der Musiker der Kapelle des Fürstprimas Esterházy, wobei der Komponist gelobt wurde:

6 Ibid, S. 416-418.

7 Vgl. dazu KAČIC, Ladislav. Die Musiker bei den Jesuiten in der Slowakei im 17. und 18. Jahrhundert. In Aurora musas nutrit. Die Jesuiten und die Kultur Mitteleuropas im 16.18. Jahrhundert. Ed. Ladislav Kačic - Svorad Zavarský. Bratislava: Slavistický ústav Jána Stanislava SAV - Teologická fakulta Trnavskej univerzity 2008, S. 215-226.

8 KILIÁN, István. Neue Daten über die Schauspielpraxis der Jesuiten in Bratislava. Ibid. (Anm. 7), S. 145-154. 
6. August 1734 Veneris. Post Vesperas proba Musicis pro Actione Majori. Aderant soli Musici Celsissimi Archiepiscopi. Probatum in cubiculo Magistri Grammaticae, data subinde collatio a Collegio in cubiculo Magistri Principiorum. Laudem tulit compositor statim in hac proba.

Der von den Mitgliedern der Fürstbischöflichen Kapelle gelobte Komponist war der damalige Student des 3. Jahres der Philosophie (Metaphysik) in Tyrnau Christian Leibetzeder SJ (geb. um 1710), später Mitglied des Jesuitenordens. ${ }^{9}$ Es sind eigentlich zwei Tatsachen an diesen und folgenden Angaben interessant, nämlich dass

1/ ein Musiker (Komponist) aus Tyrnau für die Zusammenarbeit an einem Schuldrama in Preßburg berufen wurde, und

2/ ausschließlich Musiker der Fürstbischöflichen Kapelle spielten, unter denen mehrere ausgezeichnete Musiker, ja auch Komponisten waren (J. M. Schenauer, J. Umstatt, L. Carl u. a.).

Die Erklärung ist jedoch sehr einfach: die Zusammenarbeit mit dem Komponisten war immer völlig in der Kompetenz des jeweiligen Pädagogen, Autors und zugleich Regisseurs des Dramas.

Am nächsten Tag (7.8.1734) fand die Generalprobe (ohne Musik) statt, es wurden Tänze (Ballette) produziert, die Fürstliche Musik hatte am 8.8. eine selbständige Probe im Refektorium in Anwesenheit des Fürstbischofs E. Esterházy (!):

7. August 1734. Sabbati. Scholae post Litanias. Proba Generalis Actionis Majoris. Assedit consvetae Censorum mensae Reverendissimus Pater Rector, consultores tres. Pater item Praefectus. Aderat etiam et consedit Pater Procurator Tyrnaviae, qvi cum Actionem non posset, Probam saltem spectare voluit. Musica non probata. Producti saltus et unum intermediorum tantum se ostendit. 8. Augusti Probata musica 2-dum coram ipso Celsissimo in refectorio nostro.

Am 9. August 1734 wurden die Intermedien (d. h. Prologus, Chorus I-III, Epilogus) probiert:

9. Augusti 1734 Lunae. Scholae tota die. Litaniae, media quarta Reverendus Pater Rector accepto uno consultorum cum Patre Praefecto probarunt intermedia.

11. August 1734. Mercurii Scholae invitati spectatores pro Comoedia et spectatores moniti a Praefecto Praemiferi.

Die Aufführung des Dramas fand vor dem Fürstprimas Esterházy am 12. August 1734 in Anwesenheit von J. Pálffy und einiger anderen Adeligen statt:

9 Über P. Christian Leibetzeder SJ siehe näher KIM-SZACSVAI, Katalin. Musik bei den Tyrnauer Jesuiten im 18. Jahrhundert. In Aurora musas nutrit (wie Anm. 7), S. 237. Leibetzeder stammte aus der Steiermark und trat dem Jesuitenorden 1730 bei. 
12. August 1734 [...] Media 4ta incepit Actio Major, duravit fere usque ad octavam. Argumentum erat Bajazetes a Tamerlane bello captus, ferreo inclusus. Titulus: Ludus Fortunae in Bajazete Turcorum Imperatore. Spectatores erant celsissimus Excellentissimus Mareschallus Joannes Palffi, aliquot comites et alii Magnates. Foeminis indultum interesse.[...]

In den Annuae litterae ist über diese ca. vier Stunden dauernde Vorstellung jedoch eine etwas andere, bis jetzt nicht genug reflektierte Anmerkung zu lesen:

„Juventus Gymnasii salutaverit Celsissimum Primatem, suum Maecenatem in suburbano Collegii Tusculo, sub epuli finem triclinio in theatrum verso: 4 inferiores magistri in scenam eduxerunt Ludus Fortunae Bajazete a Tamerlane devictus." $" 10$

Das bedeutet, dass dieses Drama nicht im Theatersaal des Kollegiums beim Hl. Salvator oder in der Jesuitenkirche, sondern im Rekreationshaus der Jesuiten (Tusculum) hinter den Stadtmauern (wahrscheinlich in der heutigen Spitalgasse, nahe der Kirche des hl. Ladislaus) aufgeführt wurde, und zwar so, dass das Refektorium nach dem feierlichen Mittagessen zum Theatrum umgebaut wurde. Welche von diesen Angaben richtig ist, wissen wir nicht, wenn aber die Aufführung tatsächlich im Rekreationshaus stattfand, wäre es schon der dritte Platz im Inneren (nach dem Jesuitenkollegium und der Salvator-Kirche), an welchem die Preßburger Jesuiten ihre Schuldramen aufführten.

Solche prachtvollen, 4 bis 5 Stunden dauernden Schuldramenvorstellungen waren in der Zeit von Primas E. Esterházy sicher keine Seltenheit. Im Drama Hercules Christianus sive Stephanus Dobo (Preßburg 1929) ${ }^{11}$ wurde sogar der Choreograph (,compositor et instructor saltum") Johann Georg Fridrich aus Wien engagiert. ${ }^{12}$ (Das war auch das Verdienst von E. Esterházy.) Der Chronist des Preßburger Kollegiums bemerkte dazu, dass das Stück dem Publikum gerade „praeter Saltus a Viennensi chorago instructos“" sehr gefiel. ${ }^{13}$ Auch später wurden Tanzmeister zur Zusammenarbeit an den Pressburger Jesuitendramen berufen. So hat an den Schuldramen Alexis (1756) und Ajax et Teucer (1757) der heimische Tanzpädagoge (,instructor saltum“) Karol Plachý zusammengearbeitet, der auch die Choreographie zum Drama Nehemias (Tyrnau 1761) verfasste.

10 STAUD, Géza, op. cit., Bd. I, S. 413.

11 Ebenda, S. 404, sowie die Edition der Perioche in ungarischer Sprache (die Perioche wurde diesmal selbständig in Latein, Deutsch und Magyarisch herausgegeben) Keresztyén Hercules avagy Dobó István (1729) siehe in Régi Magyar drámai emlékek XVIII. század 4/2. Jezsuita iskoladrámák. Ed. Imre Varga. Budapest: Akadémia kiadó - Argumentum kiadó 1995, S. 1078-1086. Das Stück wurde auch mit Musik (Gesang) und Tanz (Ballettmusik) verfasst (,His accedunt Musici, Milites, Saltatores, Nuncii \&c.“).

12 Vgl. KAČIC, Ladislav. Die Musik der Jesuitendramen in der Slowakei, op. cit., S. 319-334.

13 STAUD, Géza, op. cit., Bd. I, S. 404. 
Der Erzbischof Esterházy (seine Amtsperiode war 1725-1745), der mit den Jesuiten besonders enge Kontakte pflegte, war nicht nur ein großer Mäzen der bildenden Kunst und Architektur (für ihn arbeiteten solche berühmte Künstler wie z. B. G. R. Donner oder A. Galli-Bibiena), sondern auch der Musik. Seine Kapelle gehörte bestimmt zu den besten Musikensembles, die je in Preßburg wirkten; sie ist durchaus mit der berühmten Batthyányischen Kapelle aus der 2. Hälfte des 18. Jahrhunderts vergleichbar (diese Problematik ist bis heute fast unbekannt, bzw. unerforscht). Die Kapelle bestand aus ungefähr je 15 Musikern. Die prominentesten oder am längsten dienenden Mitglieder der Kapelle waren: Johann Matthias Schenauer, Friedrich Sebastian Syhn, Leopold Carl, Joseph Umstatt, ${ }^{14}$ der Kaiserliche Hofgeiger Johann Otto Rossetter und sein Sohn Johann Killian, Joseph Hilverding, Angelo Cavallari, Giacomo Calandro, ${ }^{15}$ die Trompeter Alexander Agner, Gottfried Fink, Johann Paul Nabicht u. a., mehrere von ihnen waren auch Komponisten (Schenauer, Umstatt, Carl, aber auch P. Beer u. a.). Diese Privatkapelle Esterházys hatte außer den Streichern ständig je 2 Trompeter, 2 Waldhornisten, 2 Fagottisten, von anderen Blasinstrumenten wurde die Querflöte, regelmäßig 2 Oboen usw. besetzt. (Insgesamt waren es zwischen 1725-1745 ca. 40 Musiker!) Diese Musiker haben - in Zusammenarbeit mit den Sängern des Jesuitenkollegiums und wahrscheinlich auch mit anderen Vokalsolisten (vor allem von der St. Martin-Kirche) u. a. auch mehrere Oratorien in der Jesuiten Salvator-Kirche aufgeführt. In der bisherigen Literatur sind nur zwei solche Stücke angeführt: ${ }^{16}$

\section{1/ Würckung der schuldigen Pflicht (1727),}

\section{2/ Die Beschuldigte Unschuld (1728)}

[= Captabunt in animam Justi, \& Sanguinem innocentem condemnabuunt: Psal 93. v.21. Die beschuldigte Unschuld. Oder Der sechsmal vor das Gericht gefuehrt, geurtheilt und endlich zum Todt verdamte Welt=Erlöser (1728)],

wobei es sich um keine Schuldramen, wie es G. Staud anführt, ${ }^{17}$ sondern um ,gewöhnliche" Oratorien handelt.

14 Näher über ihn siehe Joseph Umstatt: Concerti per violino (Ed. Ladislav Kačic). Bratislava: Vysoká škola múzických umení 2013.

15 Über diesen ausgezeichneten italienischen Geiger, der vorher u. a. im Teatro Capranica in Rom sowie in anderen italienischen Städten und nachher als Konzertmeister der G. B. Locatellis Operngesellschaft in Prag wirkte, vgl. JONÁŠOVÁ, Milada. Italské operní árie v repertoáru kưru katedrály sv. Vita. Sehlingova éra 1737-1756. Hudební věda, 3-4/2001, S. 283, sowie AMORE, Adriano: Giacomo Calandro, un violinista del '700. In Le Vie della Musica, 2007, Anno VIII, no. 46, S. 38-39, (bzw. elektronisch als) „Giacomo Calandro“ (http://www.terredeigambacorta.org).

16 PUKÁNSZKY, Béla. Geschichte des deutschsprachigen Schrifttums in Ungarn. Münster: Aschendorffsche Verlagsbuchhandlung 1931, S. 369. STAUD, Géza, op. cit., S. 400-401 und 403. KAČIC, Ladislav. Die Musik der Jesuitendramen, op.cit., S. 323.

STAUD, Géza, op. cit., S. 400-401, bzw. 403. 
Beide Kompositionen stammen vom Autoren-Tandem Friedrich Syhn (Librettist, der allerdings auch Musiker, Mitglied der Esterházyschen Kapelle und zugleich „Cancellista“ an Esterházys Hof war) und Johann Matthias Schenauer (Cammer-Musicus). ${ }^{18}$ In der bisherigen Literatur fehlen aber folgende vier Preßburger Oratorien, die ebenfalls vom Librettisten Syhn und Komponisten Schenauer stammen:

3/ Das Leben in dem Todt oder Das durch die Goettliche Liebe des Leidens und sterbenden Heylands von Hoelle, Suend und Todt erloeßte Menschliche Geschlecht $[. .$.$] gesungener vorgestellet in einem Oratorio [...] (1726);$

4/ Memoria justi cum laudibus: oder Denck= und Ehren-Wahl: Durch die drey Kraefften der Seele als Bedaechtnuß, Verstand und Wille [...] zu Lob und schuldiger Ehre Dem Wunderthaetigen Blut=Zeugen S. Joanni von Nepomuck. In einem Oratorio gesungener vorgestellet (1726);

5/ Freuden=Streit: Oder Die Music in der Music. Als Der Hochwürdigst=Hochgebohrne des Heil Römis. Reichs Fürst und Herr Herr Emericus Aus deren Grafen Eszterhazy [...] sein den fünfften Novembris glückseeligst einfalenndes Nahmens =Fest Annô 1728. wiederhollete [...] (1728);

6/ Ascensiones in corde suo disposuit: in valle lacrymarum. Psal. 83. Vers. 6.27. Sechsfacher Anstoß Des Zum Berg Calvariae im Geist aufsteigenden Wanders=Mann, [...] Das ist Sechsfache Frucht Der Betrachtung des Leidens und Sterbens Christi [...] in eben so vielen Oratoriis [...] zu Preßburg...Musicalisch vorgestellet im Jahr 1729 (1729).

Es sind, leider, nur Textbücher von diesen sechs Kompositionen überliefert, aber keine Musik. ${ }^{19}$ Man kann sich jedoch trotzdem eine gute Vorstellung von diesen Oratorien machen.

Es handelt sich entweder um Oratorien des Sepolcro-Typus - Das Leben in dem Todt (1726), Die Beschuldigte Unschuld (1728), Ascensiones in corde suo... Sechsfacher Anstoß (1729), oder um das Martyrium des hl. Johann von Nepomuk - Memoria justi cum laudibus: oder Denck= und Ehren-Wahl (1726), oder aber um typische Gelegenheitskompositionen zum Namenstag des Fürstbischofs - Würckung der schuldigen Pflicht (1727) und Freuden=Streit: Oder Die Music in der Music (1728).

18 PUKÁNSZKY, Béla, op. cit., S. 217-218 vermutete, dass Syhn und Schenauer aus Wien stammten, beide waren jedoch Mitglieder der Kapelle E. Esterházys (mehr darüber in einer Studie über diese Kapelle, in Vorb.).

19 Die Beschuldigte Unschuld und Ascensiones in corde suo disposuit - Sechsfacher Anstoß wurden auch von F. A. Míča vertont und zwischen 1729-1736 mehrmals in Olmütz und Brünn aufgeführt. Vgl. PERUTKOVÁ, Jana. František Antonín Miča ve službách hraběte Questenberga a italská opera v Jaroměřicích. Praha: KLP 2011, S. 211 und 457-458. 
In Wirklichkeit handelt es sich also um keine jesuitischen Schuldramen, sondern um reguläre Oratorien. Die Mehrzahl von ihnen - bestimmt alle drei Sepolcri - wurden in der Preßburger Jesuitenkirche aufgeführt, alle diese Stücke haben aber keine Struktur eines Jesuitendramas (Prologus - Actus I - Chorus I - Actus II - Chorus II - Actus III - Epilogus), sondern jene des Oratoriums. Die Beschuldigte Unschuld (1728) wurde in der Jesuitenkirche des Allerheiligsten Erlösers in Preßburg - wie es am Titelblatt steht - ,durch sechs Freytäge der Fasten, in eben so vielen Oratoriis zu Ehren des Leidenden Heylands" aufgeführt.

Im Fürstbischöflichen Archiv sind mehrere interessanten Angaben zu diesen und anderen solchen Aufführungen zu finden, z. B. die Zahlung von 60 Fl. an den Preßburger Buchdrucker J. P. Royer für den Druck der Libretti von 6 Oratorien (1728) (Fig. 1a, 1b), eine Zahlung für das „Abschreiben der Musicalien“ zu 6 Oratorien (1728) an den Musiker Joseph Durant (6 Fl), oder eine Zahlung für ,geschriebene Musicalien“ zu einem ,in honorem St. Joannis Nepomuceni“ aufgeführten Oratorium (1729) an den Bassisten der St. Martin-Kirche Bernhard Ponnheimer, Zahlungen an einzelne Musikern (vor allem aus der St. MartinDomkirche und Jesuitenkirche, d. h. außer an die Mitglieder der Fürstbischöflichen Kapelle) 1728 (Fig. 2a, 2b) usw.
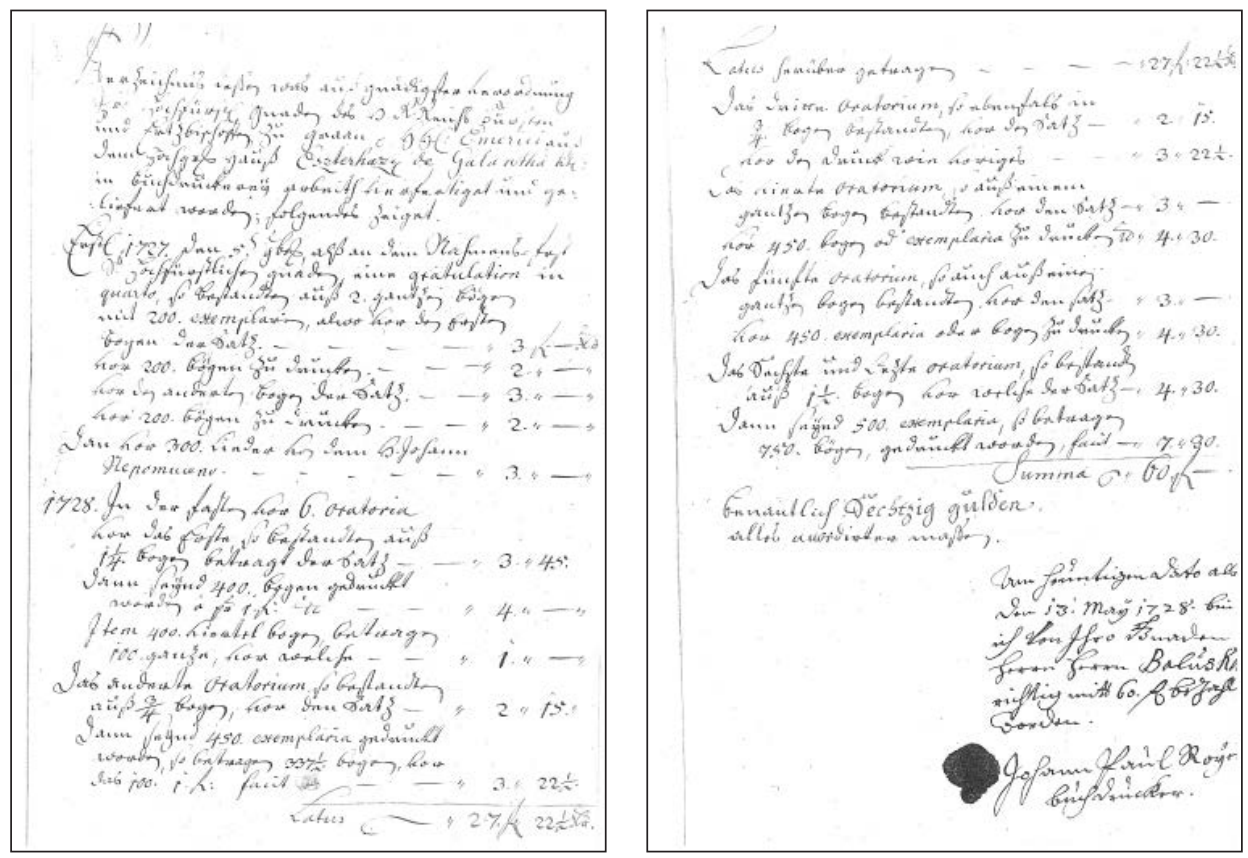

Fig. 1a, 1b 

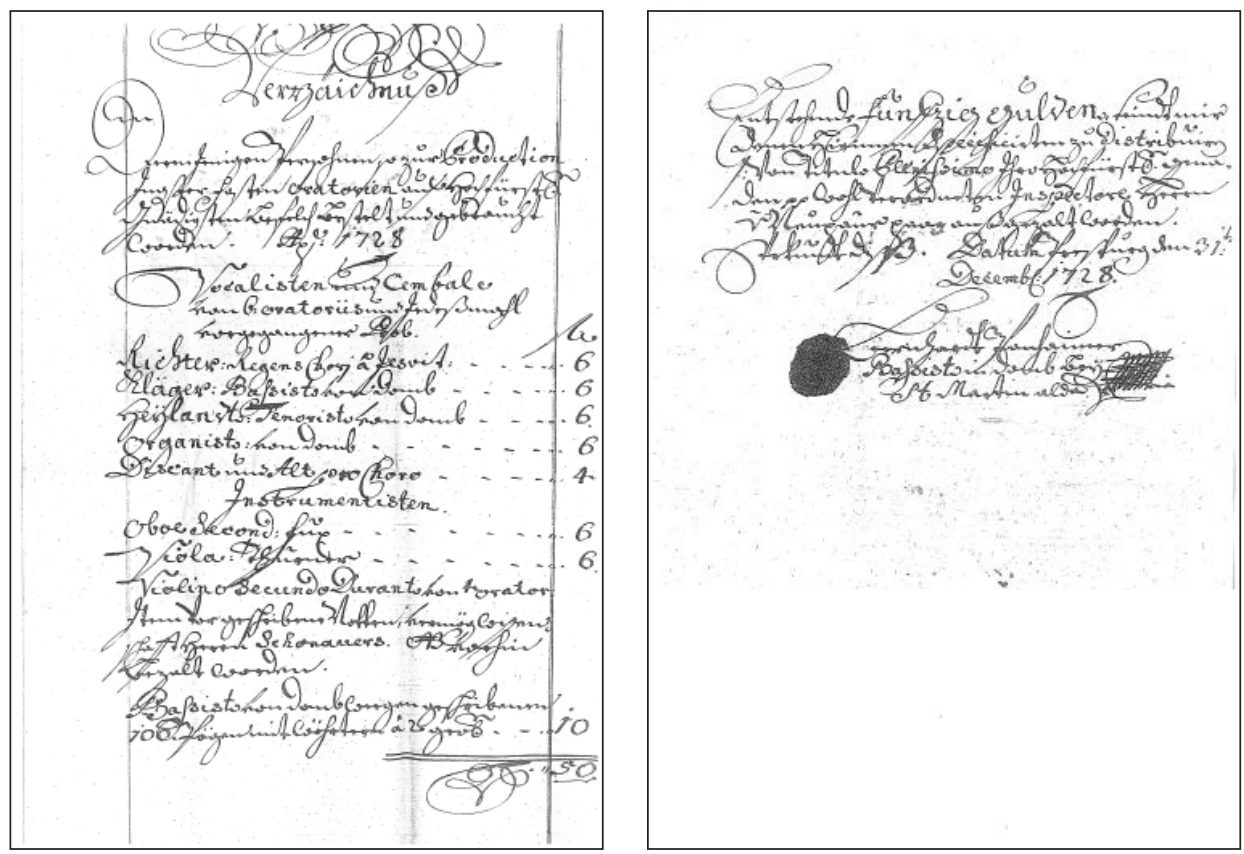

Fig. 2a, 2b

Am 19. 10. 1728 wurden verschiedene Zahlungen im Zusammenhang mit der Aufführung eines Oratoriums bei der Statue des hl. Johann von Nepomuk im Garten des Fürstbischöflichen Palais geleistet, u. a. an den Komponisten J. M. Schenauer:

„Ex Mandato Suae Celsitudinis munerari Dno. Senauer [sic!] pro commoditate revehendorum Musicorum Cardinalis Csaki, qui tempore Benedictionis Statuae S. Joannis Nepomuceni Oratorium instituerant..."

Am 31. 7. 1731 wurden 6 Kremnitzer Dukaten an den Kaiserlichen Tanzmeister („Caesareo Saltûs Magistro“) und am 7.9.1731 wiederum 70 Fl 40 x (bzw. 12 Kremnitzer Dukaten) an Schenauer im Zusammenhang mit Schuldramen gezahlt, die bei den Preßburger Jesuiten aufgeführt wurden (,Musicis, qui in Comoediis 6 . currentis apud R.R.P.P. Societatis productis servierunt").

Interessant sind aber auch die Angaben über die Gesamtkosten für eine solche Schuldramenaufführung. Die Liste vom 28. 7. 1731 („Pro Theatro ad hujas S. $J$. Collegio") führt insgesamt $460 \mathrm{Fl} 12 \mathrm{x}$ an, und zwar inklusive Dekorationen, Perioche (lateinisch und deutsch), „Honorarium Compositoris Musicae“ 12,-- Fl, die Zahlungen an die Musiker usw.; dies alles wurde vom Fürstbischof eigenhändig bestätigt. (Fig. 3a, 3b)

Aber zurück zu den Kompositionen von Schenauer und Syhn: 
Die Würckung der schuldigen Pflicht $(1727)^{20}$ ist - textlich - ein Hirtenspiel (,Schäferspiel“), in dem der gefeierte Fürstprimas Esterházy als der große Hirt Orastes (Orastes = Anagramm von „Estoras“, d. h. Esterházy) „in einem lobsingenden Chor mythologischer und bukolischer Gestalten umschwärmt wurde“. Es „singen vier durch genauiste Freündschaft verbundene Hirten."

Würckung der schuldigen Pflicht (1727) - die Besetzung nach dem Textbuch:

Fideno

Damon

Mirtillo Sohn des Damons

Silvio

Chor deren Hirten

ORASTES / Durch Buchstaben Wechsel / ESTORAS [= nicht singende Person]

Das Stück besteht ähnlich wie Die Beschuldige Unschuld aus Rezitativen, Arien (vornehmlich Arien da Capo), inklusive einer im Barock so beliebten EchoArie („Wann ich dich anjetzt befrage“, Echo: „Frage!“ usw.), einer Aria a 2 (= Duett), Arioso (,Tugend hat oft harte Wege“) und des Chors der Hirten (nicht nur zum Beschlu $\beta$, sondern auch innerhalb des Spiels), es gibt im Stück sogar eine Aria (Fideno) mit Chor „Seh’t wie uns durch Gnaden-Blicke“ Chor: „Ja, ja!“), der abschließende Chor singt „Nicht straffe grosse Hirt“ und schliesslich „Es lebe ORASTES! Im Wohlstand und Glücke“ usw.; es handelt sich also um eine typische Huldigungskomposition.

Mit unserem Thema haben jedoch vor allem zwei, bzw. drei Oratorien zu tun, die wir zum Schluss kurz vorstellen möchten:

Georg Christoph Wagenseil: Mater dolorum Das ist: die Schmerzhafte Mutter (vor 1773),

Georg Christoph Wagenseil: Oratorio per la novenna di S. Xaverio (vor 1760) und

Georg Christoph Wagenseil: Oratorio per la novenna di S. Ignatio (unkomplett).

Alle drei Werke ${ }^{21}$ wurden - im Unterschied zu den genannten Esterházyschen Oratorien - von den Musikern der Jesuitenkirche aufgeführt. (Keines davon ist

20 Textbuch in Egyetemi könyvtár, Budapest, Sign. Min. A/65.

21 Das Notenmaterial der Oratorien zum hl. Franz Xaver und hl. Ignatius ist Bestandteil einer kleinen, bis jetzt unbearbeiteten und nicht katalogisierten Musikaliensammlung der Jesuiten in Bratislava. Die meisten Stimmhefte stammen wahrscheinlich von der Hand eines professionellen Wiener Kopisten, nur die hier und da vorkommenden Dubletten wurden von den 
übrigens im Thematischen Katalog Wagenseils zu finden, diese Oratorien sind also bis heute unbekannt. ${ }^{22}$ )

Von Wagenseils Mater dolorum (1773) ${ }^{23}$ ist lediglich auch nur ein Libretto überliefert. (Fig. 4a) Ähnlich wie im Wiener Sepolcro, kommen auch in diesem Stück nur einige wenige Rollen vor:

\title{
Georg Christoph Wagenseil: Mater dolorum (1773) - die Besetzung
}

\author{
Maria die schmerzhafte Mutter Gottes, \\ Johannes der heilige Jünger Christi, \\ Joseph von Arimathia, \\ Nicodemus, \\ Das Christliche Mitleiden [eine allegorische Gestalt].
}

Das Libretto von H. Rademin wurde vorher schon von J. G. Reutter d. J. (1726) und wahrscheinlich auch von G. J. Werner (1733) ${ }^{24}$ vertont, die Preßburger Aufführung des Oratoriums Wagenseils war aber auch nicht die erste, vorher wurde diese Komposition „An der Kirchen deren Wohl-Ehrwürdigen Closter=Frauen bey St. Jacob in Wienn" (,Gedruckt bey Johann Ignatz Heyinger, Universität=Buchdruckern“) aufgeführt, ${ }^{25}$ das müsste vor 1755 sein, weil J. I. Heyinger in diesem Jahr gestorben ist. (Fig. 4b)

Die zweiteilige Komposition besteht aus Rezitativen (größtenteils EnsembleRezitativen), Arien (Da Capo, aber auch einige Strophenarien), Ariosi (z. B. jenes von Maria - „Es stirbt mein Sohn!“ - müsste sehr dramatisch sein), Duetti und abschließenden Chören aller beteiligten Personen. Der 1. Teil behandelt die Situation unter dem Kreuz, der 2. Teil die Grablegung und nachher. Der Text schöpft nicht nur aus den Evangelien (Passion), sondern auch aus dem Alten Testament (Jeremias, Threni u.s.w.). Das erste Rezitativ (Johannes) beginnt: „Es ist vollbracht, daß war sein letztes Wort..." Die abschließenden Nummern beider

Musikern der Jesuitenkirche kopiert.

Sie sind weder bei SCHOLZ-MICHELITSCH, Helga. Georg Christoph Wagenseil, Hofkomponist und Hofklaviermeister der Maria Theresia. Wien: Wilhelm Braunmüller 1980, noch in den neuesten Lexika (MGG, Grove) zu finden.

STAUD, Géza, op. cit., I, S. 450. Vorher hat G. Staud das Stück auf 1767 datiert - STAUD, Géza. A templomtér mint színház. In Irodalomtörténeti közlémények, Budapest 1983, S. 665669. Unserer Meinung nach ist diese Datierung (1767) realistischer, bzw. besser als „1773“.

$\mathrm{Zu}$ Reutter vgl. RIEDEL, Friedrich W. Die Libretto-Sammlung im Benediktinerstift Göttweig. Fontes Artis Musicae, 13, 1966, S. 452-455; zu Werners Komposition vgl. TANK, Ulrich. Studien zur Esterházyschen Hofmusik von etwa 1620 bis 1790 (Kölner Beiträge zur Musikforschung, Bd. 101). Regensburg: Gustav Bosse Verlag 1981, S. 217-218. In Werners Komposition sind nur vier Personen besetzt (Maria, Johannes, Nicodemus, Joseph von Arimathia), es fehlt die allegorische Gestalt „Das Christliche Mitleiden“. Librettos Mater dolorum (J. M. Landerer, o. J.) - Egyetemi Könyvtár, Budapest, Sign. J4º 36 Coll.4 - ist mit dieser Ausgabe praktisch identisch. 
Teile sind auch „Aria“ (für Chor) bezeichnet - 1. „Verführte Salems-Kinder“, 2. Schlu $\beta=$ Arie „Erfreue dich sündige Welt!, d. h. durchaus ein positiver Abschluss der Passionsgeschichte:

\section{Erfreue dich sündige Welt!}

Mit JESU nun liegt

verschlossen, vergraben, was alle gesündigt haben,

Der Tod wird besiegt

und Gott ist zufrieden gestellt,

Erfreue dich sündige Welt!

Die Musik Wagenseils kennen wir also, leider nicht, eine in der Pressburger Jesuitenkirche mehrmals aufgeführte Komposition des Wiener Hofkomponisten - Oratorio per la novenna di S. Xaverio - ist jedoch komplett überliefert, d. h. das komplette Notenmaterial, von einer anderen Komposition - Oratorio pre la novenna di S. Ignatio - ist wiederum nur ein Bruchteil erhalten geblieben (siehe weiter). Auch diese Oratorien sind ähnlich wie alle vorher genannten Werke in deutscher Sprache. (Preßburg war ja im 18. Jahrhundert eine „deutsche Stadt“, Slowaken waren Minderheit und Ungaren kamen nur selten vor.)
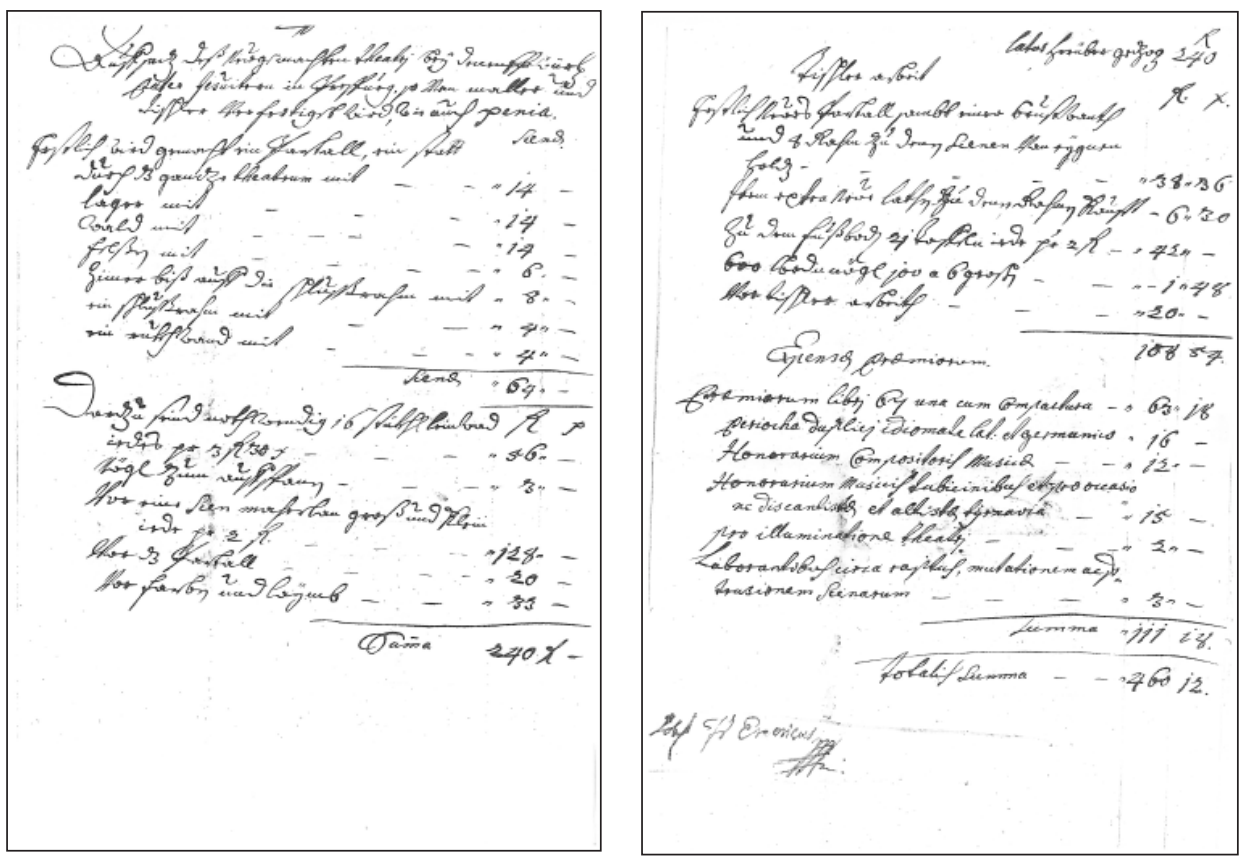

Fig. $3 a, 3 b$ 

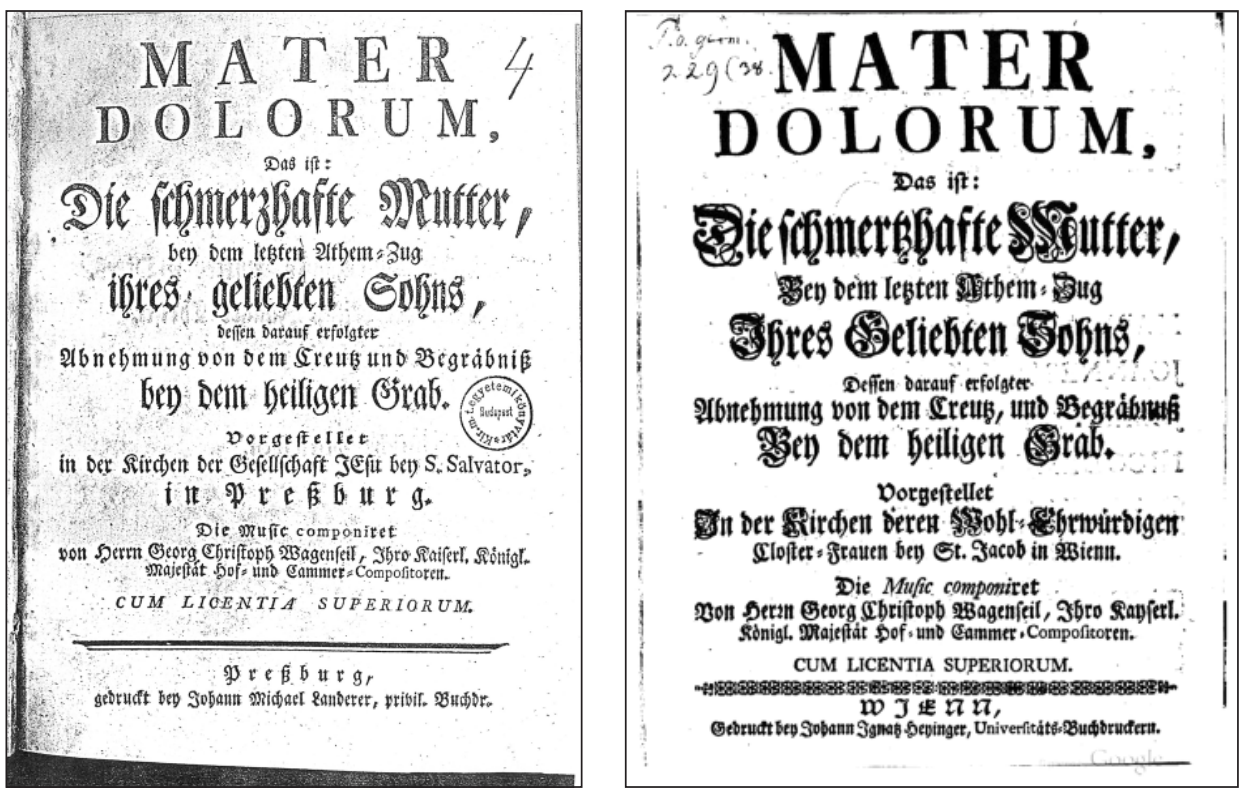

Fig. $4 \mathrm{a}, 4 \mathrm{~b}$
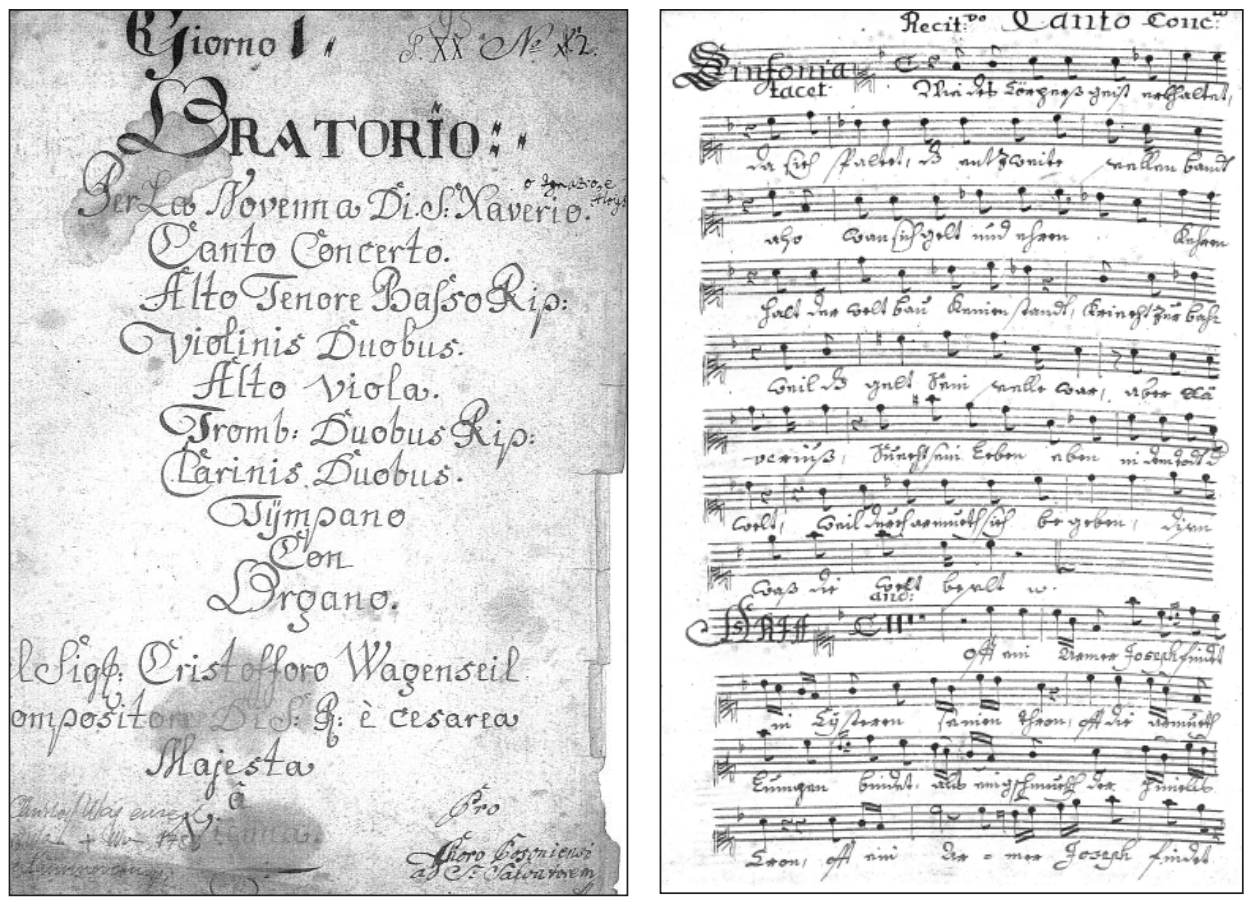

Fig. $5 \mathrm{a}, 5 \mathrm{~b}$ 
Wagenseils Oratorio per la novenna di S. Xaverio (Fig. 5a, 5b) ist in Wirklichkeit mehr ein an vier Tagen der Novene zum hl. Franz Xaver aufgeführter Kantaten-Zyklus (Giorno I - Giorno IV) als ein Oratorium. Es kommen nämlich keine konkreten Personen im Libretto vor, jede Komposition besteht aus einem instrumentalen Eröffnungssatz (Sinfonia im Giorno I und Giorno IV, bzw. Introduzzione im Giorno II und Giorno III), nach dem Rezitativ folgt eine Arie oder ein Duetto und der Abschlusschor. Die einzelnen vier Kompositionen sind also kurz, aber die instrumentale Besetzung ist festlich, mit Trompeten, im ersten und letzten Stück kommen noch zwei Posaunen dazu.

\section{Georg Christoph Wagenseil: Oratorio per la novenna di S. Xaverio}

Die Struktur der vier Teile der Komposition (Giorno I - Giorno IV) ist gleich:

Sinfonia (bzw. Introduzzione im Giorno II und Giorno III)

Recitativo

Aria (oder Duetto im Giorno II)

Chorus (Coro)

Der Text der ganzen Komposition ist an sich sehr seltsam, mystisch, keinesfalls dramatisch (weder in den Rezitativen noch in den Arien) und typisch für ein Oratorium. In den einzelnen Nummern des ganzen Stückes werden einige hervorragende Eigenschaften und Tugenden des hl. Franz hervorgehoben, sein Abkehr von Reichtum, Welt, Geld und Ehren zum Himmel, zu Armut und zum Dienst an jenen, die es brauchen (Armen usw.), wie auch seine totale Hingabe an den Willen Gottes. Im Kern wird also die typische Xaverianische Spiritualität und Frömmigkeit des hl. Franz hervorgehoben, es geht also nicht um die typische jesuitische Apotheose (seit G. Kapsbergers Apotheosis sive Consecratio SS. Ignatii et Francisci Xaverii, 1622 ) des Heiligen. Kompositorisch bringt die Komposition eine gute, qualitätsvolle, eine typische um die Mitte des 18. Jahrhunderts entstandene Musik, beginnend mit den einsätzigen Eröffnungsinstrumentalsätzen über die Rezitative und Arien da capo (alle im langsameren Tempi) bis zu den abschließenden festlichen (mit Trompeten, bzw. auch mit den für den österreichisch-mitteleuropäischen Raum typischen Posaunen), jedoch strukturell sehr einfachen Chören.

Eine ähnliche Struktur hat auch Wagenseils Oratorio pre la novenna di S. Ignatio, das aber nicht komplett, sondern nur für Giorno I erhalten geblieben ist. Die Komposition besteht auch aus einer einleitenden einsätzigen Sinfonia, es folgt Recitativo („Erstaunliche Liebe“), Aria („Wie die reine Flamm’ der Sternen“) und Chorus („Eÿlet, eÿlet erhitzt"); Sinfonia und Abschlusschor sind mit Trompeten besetzt (die am Titelblatt angeführten Stimmhefte der Posaunen fehlen). Es handelt sich also auch mehr um eine „Kantate“ als um ein Oratorium. Am Titelblatt (verso) gibt es eine Bleistift-Anmerkung über die Aufführung des Werkes in der Preßburger Jesuitenkirche: „1764“. Ähnliche Anmerkungen sind auch im Material des Oratorios per la novenna di S. Xaverio zu finden: Giorno I- „1760“ und „1763“ Giorno III - „1762“ und Giorno IV - „1762“, „1763“, „1764“; d. h. 
alle diese Werke wurden vor allem in den 60er-Jahren des 18. Jahrhunderts aufgeführt, sehr wahrscheinlich nicht zusammen, sondern selbständig, als „Kantaten“.

Beide letztgenannten Oratorien stammen aus ca. derselben Zeit wie alle drei bis jetzt bekannten italienischen Oratorien Wagenseils: Gioas, re di Giuda (1755), La rendenzione (1755) und Il roveto di Mose (1756). ${ }^{26}$ Musikalisch passen also Oratorio per la novenna di Sancto Xaverio und Oratorio pre la novenna si S. Ignatio genau in diese Schaffensperiode Wagenseils und bringen Kompositionen von guter Qualität im Stil aus der Wende des Spätbarocks zur Frühklassik.

Den konkreten Kontext der wiederholten Aufführungen dieser Kompositionen in der Preßburger Jesuitenkirche in den Jahren ca. 1760-1765 kennen wir nicht, d. h. ob sie im Rahmen des Gottesdienstes oder bei einer anderen Gelegenheit aufgeführt wurden, es war aber bestimmt auch eine Festrede (Predigt, bzw. ein Exhortatio u. ä.) dabei.

Kurz zusammenfassend: Bei den Preßburger Jesuiten wurden im 18. Jahrhundert nicht nur die gewöhnlichen, typischen Schuldramen, sondern auch Oratorien aufgeführt. Die vor allem in den 20er und 30er Jahren des 18. Jahrhunderts regelmäßigen Oratorien-Aufführungen in der Preßburger Jesuitenkirche (unter Mitwirkung der Kapelle des Fürstbischofs Esterházy) waren eigentlich eine Spezifik, und keinesfalls typisch für alle Jesuitenkollegien in der Slowakei. Damit wurde das Preßburger Kollegium mit der Salvator-Kirche musikalisch die bedeutendste Jesuiten-Institution in der Slowakei. Ein klarer Beweis dafür sind die bis jetzt unbekannten Quellen - Libretti der Kompositionen von J. M. Schenauer, sowie das überlieferte Notenmaterial aus den Oratorien G. Ch. Wagenseils.

Ladislav Kačic (ladislav.kacic@savba.sk), Slovenská akadémia vied.

\section{ABSTRACT \\ SCHOOL DRAMAS AND ORATORIOS BY THE PRESSBURG JESUITS IN 18TH CENTURY}

The oratorio was certainly not one of the favorite musical genres of the Jesuits in the $17^{\text {th }}-18$ century. The Pressburg Jesuits, however, have also performed oratorios in the $18^{\text {th }}$ century, except their typical school dramas. The "golden age" of the oratorio in Pressburg were the 20s and 30s of the $18^{\text {th }}$ century, i. e. the period of office of the Prince Primate Emericus Esterházy. At that time, there were performed 1-2 oratorios per year dedicated to the Primate by the libretits F. Syhn and by composer J. M. Schenauer in the Bratislava Jesuit Church. We know of them unfortunately only the text books. The Pressburg Jesuits have also worked with the court composer G. Ch. Wagenseil: from the oratorio Mater dolorum (1773) has only been handed a libretto, a yet unknown Oratorio per la Novenna di S. Xaverio (1750), however, is completely preserved.

\section{Key words}

Bratislava (Pressburg), Jesuits, the $18^{\text {th }}$ century, school drama, oratorio, Wagenseil, new sources 


\section{Bibliography}

AMORE, Adriano: Giacomo Calandro, un violinista del '700. In Le Vie della Musica, 2007, Anno VIII, no. 46, S. 38-39, (bzw. elektronisch als) „Giacomo Calandro“ (http://www.terredeigambacorta.org).

Aurora musas nutrit: Die Jesuiten und die Kultur Mitteleuropas im 16.-18. Jahrhundert. Ladislav Kačic - Svorad Zavarský (eds.). Bratislava: Slavistický ústav Jána Stanislava SAV - Teologická fakulta Trnavskej univerzity, 2008.

KRAPKA, Emil SJ - MIKULA, Vojtech SJ. Dejiny Spoločnosti Ježišovej na Slovensku 15611988. 1. Ed. Cambridge, On. (Canada): Dobrá kniha, 1990.

JONÁŠOVÁ, Milada. Italské operni árie v repertoáru kůru katedrály sv. Vita. Sehlingova éra 1737-1756. Hudební věda, 3-4/2001.

KAČIC, Ladislav. Die Musik der Jesuitendramen in der Slowakei (1600-1773): Ein Beitrag zur Geschichte der Provincia Austriae SJ. In Ethnologische, historische und systematische Musikwissenschaft (Oskár Elschek zum 65. Geburtstag). Franz Födermayr - Ladislav Burlas (eds.). Bratislava: ASCO Art and Science, 1998.

KRAMER, Waltraute. Die Musik im Wiener Jesuitendrama von 1677 bis 1767 (Diss.), Wien, 1965.

Libretto Mater dolorum (J. M. Landerer, o. J.) - Egyetemi Könyvtár, Budapest, Sign. J4 36 Coll.4. / Bayeriche Staatsbibliothek, München, Sign. P.o.germ. 229/38.

PERUTKOVÁ, Jana. František Antonín Mǐca ve službách hraběte Questenberga a italská opera v Jaroměricich. Praha: KLP, 2011.

PUKÁNSZKY, Béla. Geschichte des deutschsprachigen Schrifttums in Ungarn. Münster: Aschendorffsche Verlagsbuchhandlung, 1931.

Régi Magyar drámai emlékek XVIII. század 4/2. Jezsuita iskoladrámák. Imre Varga (ed.). Budapest: Akadémia kiadó - Argumentum kiadó, 1995.

RIEDEL, Friedrich W. Die Libretto-Sammlung im Benediktinerstift Göttweig. Fontes Artis Musicae, 13, 1966.

SCHOLZ-MICHELITSCH, Helga. Georg Christoph Wagenseil, Hofkomponist und Hofklaviermeister der Maria Theresia. Wien: Wilhelm Braunmüller, 1980.

STAUD, Géza. A Maygarországi jezsuita iskolai színjátékok forrásai (Fontes ludorum scenicorum in scholis S. J. Hungariae), Bd. I, Budapest: Magyar Tudományos Akadémia, 1984.

STAUD, Géza. A templomtér mint színház. In Irodalomtörténeti közlémények, Budapest, 1983.

TANK, Ulrich. Studien zur Esterházyschen Hofmusik von etwa 1620 bis 1790 (Kölner Beiträge zur Musikforschung, Bd. 101). Regensburg: Gustav Bosse Verlag, 1981.

UMSTATT, Joseph: Concerti per violino (Ed. Ladislav Kačic). Bratislava: Vysoká škola múzických umení, 2013. 